Cavendish-HEP-03/15

FSU-HEP-030725

\title{
Next-to-next-to-leading-order soft-gluon corrections in direct photon production
}

\author{
Nikolaos Kidonakis \\ Cavendish Laboratory, University of Cambridge, \\ Madingley Road, Cambridge CB3 OHE, UK \\ J.F. Owens \\ Physics Department, Florida State University, \\ Tallahassee, FL 32306-4350, USA
}

\begin{abstract}
Previous work on soft-gluon resummation for direct photon production is extended to include additional subleading logarithmic terms through $\mathcal{O}\left(\alpha \alpha_{s}^{3}\right)$ and some representative comparisons are made to experimental results from the E-706 and UA-6 Collaborations. The additional terms are small in magnitude, indicating good convergence properties to the level of accuracy calculated. The scale dependence remains much smaller than that of the next-to-leading-order calculation.
\end{abstract}




\section{INTRODUCTION}

Direct photon production is widely recognized as a process that is potentially important in determinations of the gluon distribution function. The next-to-leading-order (NLO) cross section for direct photon production has been given in Refs. [1, 2]. The role of higher-order soft-gluon corrections has also been addressed more recently. Threshold resummation studies for direct photon production have appeared in Refs. [3, 4, 5, 6] while a joint threshold and transverse momentum resummation formalism has been given in Ref. [7].

In a previous paper [5] we presented analytical and numerical results for the next-to-nextto-leading-order (NNLO) next-to-next-to-leading-logarithm (NNLL) soft-gluon corrections for direct photon production. Here we increase the accuracy of our previous calculation by including additional subleading soft corrections. Our approach follows Ref. 8] which in turn is based on and extends previous work on threshold resummation [3, 9, 10, 11, 12, 13].

\section{NNLO SOFT CORRECTIONS}

At lowest order, the parton-parton scattering subprocesses are $q\left(p_{a}\right)+g\left(p_{b}\right) \rightarrow \gamma\left(p_{\gamma}\right)+q\left(p_{J}\right)$ and $q\left(p_{a}\right)+\bar{q}\left(p_{b}\right) \rightarrow \gamma\left(p_{\gamma}\right)+g\left(p_{J}\right)$. We define the Mandelstam invariants $s=\left(p_{a}+p_{b}\right)^{2}$, $t=\left(p_{a}-p_{\gamma}\right)^{2}$, and $u=\left(p_{b}-p_{\gamma}\right)^{2}$, which satisfy $s_{4} \equiv s+t+u=0$ at threshold. Note that the photon transverse momentum is $p_{T}=(t u / s)^{1 / 2}$. Here we calculate the cross section $E_{\gamma} d^{3} \sigma / d^{3} p_{\gamma}$ in single-particle-inclusive kinematics in the $\overline{\mathrm{MS}}$ scheme. The soft corrections to the cross section appear in the form of plus distributions

$$
\mathcal{D}_{l}\left(s_{4}\right) \equiv\left[\frac{\ln ^{l}\left(s_{4} / p_{T}^{2}\right)}{s_{4}}\right]_{+}
$$

with $l \leq 2 n-1$ at $n$th order in $\alpha_{s}$ beyond the leading order, while the virtual corrections appear in $\delta\left(s_{4}\right)$ terms.

We begin with the NLO soft and virtual corrections in the $\overline{\mathrm{MS}}$ scheme. A somewhat different notation from that used in Ref. [5] has been adopted here, so the previously calculated terms are repeated here, as well. The corrections to the parton-level cross section, $\hat{\sigma}$, can be written for either subprocess as

$$
E_{\gamma} \frac{d^{3} \hat{\sigma}_{f_{i} f_{j}}^{(1)}}{d^{3} p_{\gamma}}=\sigma_{f_{i} f_{j}}^{B} \frac{\alpha_{s}\left(\mu_{R}^{2}\right)}{\pi}\left\{c_{3}^{f_{i} f_{j}} \mathcal{D}_{1}\left(s_{4}\right)+c_{2}^{f_{i} f_{j}} \mathcal{D}_{0}\left(s_{4}\right)+c_{1}^{f_{i} f_{j}} \delta\left(s_{4}\right)\right\},
$$


where $\mu_{R}$ is the renormalization scale, and the Born terms are given by

$$
\sigma_{q \bar{q}}^{B}=\frac{2 C_{F}}{N_{c}} \frac{\alpha \alpha_{s}}{s} e_{q}^{2}\left(\frac{t}{u}+\frac{u}{t}\right), \quad \sigma_{q g}^{B}=-\frac{1}{N_{c}} \frac{\alpha \alpha_{s}}{s} e_{q}^{2}\left(\frac{s}{t}+\frac{t}{s}\right)
$$

where $e_{q}$ is the charge of a quark of type $q$, and $C_{F}=\left(N_{c}^{2}-1\right) /\left(2 N_{c}\right)$ with $N_{c}=3$ the number of colors. Also $c_{3}^{q \bar{q}}=4 C_{F}-C_{A}, c_{3}^{q g}=C_{F}+2 C_{A}$,

$$
c_{2}^{q \bar{q}}=-\frac{\beta_{0}}{4}-2 C_{F} \ln \left(\frac{\mu_{F}^{2}}{p_{T}^{2}}\right), \quad c_{2}^{q g}=-\frac{3}{4} C_{F}-\left(C_{F}+C_{A}\right) \ln \left(\frac{\mu_{F}^{2}}{p_{T}^{2}}\right)
$$

where $\mu_{F}$ is the factorization scale, $C_{A}=N_{c}$, and $\beta_{0}=\left(11 C_{A}-2 n_{f}\right) / 3$, with $n_{f}$ the number of quark flavors. We also define for use below $T_{2}^{q \bar{q}}=-\left(\beta_{0} / 4\right)+2 C_{F} \ln \left(p_{T}^{2} / s\right)$ and $T_{2}^{q g}=$ $-(3 / 4) C_{F}+\left(C_{F}+C_{A}\right) \ln \left(p_{T}^{2} / s\right)$. Finally we write $c_{1}^{f_{i} f_{j}}=c_{1}^{\mu f_{i} f_{j}}+T_{1}^{f_{i} f_{j}}$. For $q \bar{q} \rightarrow \gamma g$ we have

$$
c_{1}^{\mu q \bar{q}}=C_{F}\left[-\frac{3}{2}-\ln \left(\frac{p_{T}^{2}}{s}\right)\right] \ln \left(\frac{\mu_{F}^{2}}{s}\right)+\frac{\beta_{0}}{4} \ln \left(\frac{\mu_{R}^{2}}{s}\right),
$$

and $T_{1}^{q \bar{q}}=C_{F}\left[3 / 2+\ln \left(p_{T}^{2} / s\right)\right] \ln \left(p_{T}^{2} / s\right)-\left(\beta_{0} / 4\right) \ln \left(\mu_{R}^{2} / s\right)+c_{1}^{\prime q \bar{q}}$ where $c_{1}^{\prime q \bar{q}}$ is defined in Eq. (3.11) of Ref. [5]. For $q g \rightarrow \gamma q$ we have

$$
c_{1}^{\mu q g}=\left[-\frac{\beta_{0}}{4}-\frac{3}{4} C_{F}-C_{F} \ln \left(\frac{-t}{s}\right)-C_{A} \ln \left(\frac{-u}{s}\right)\right] \ln \left(\frac{\mu_{F}^{2}}{s}\right)+\frac{\beta_{0}}{4} \ln \left(\frac{\mu_{R}^{2}}{s}\right)
$$

and $T_{1}^{q g}=\left[3 C_{F} / 4+C_{F} \ln (-t / s)+C_{A} \ln (-u / s)\right] \ln \left(p_{T}^{2} / s\right)-\left(\beta_{0} / 4\right) \ln \left(\mu_{R}^{2} / p_{T}^{2}\right)+c_{1}^{\prime q g}$ where $c_{1}^{\prime q g}$ is defined in Eq. (3.8) of Ref. [5]].

Note that the NLO $c_{i}$ coefficients have also been presented in Ref. [5]. The notation for $c_{3}$ and $c_{2}$ is the same as before, while the notation for splitting $c_{1}$ into $c_{1}^{\mu}$ and $T_{1}$ terms for each subprocess is new and useful in presenting the NNLO expressions below.

Next, we turn to the NNLO soft and virtual corrections in the $\overline{\mathrm{MS}}$ scheme. These corrections can be written for either channel as

$$
E_{\gamma} \frac{d^{3} \hat{\sigma}_{f_{i} f_{j}}^{(2)}}{d^{3} p_{\gamma}}=\sigma_{f_{i} f_{j}}^{B} \frac{\alpha_{s}^{2}\left(\mu_{R}^{2}\right)}{\pi^{2}}{\hat{\sigma^{\prime}}}_{f_{i} f_{j}}^{(2)} .
$$

For the $q \bar{q} \rightarrow \gamma g$ process we have

$$
\begin{aligned}
& {\hat{\sigma^{\prime}}}_{q \bar{q}}^{(2)}=\frac{1}{2}\left(c_{3}^{q \bar{q}}\right)^{2} \mathcal{D}_{3}\left(s_{4}\right)+\left[\frac{3}{2} c_{3}^{q \bar{q}} c_{2}^{q \bar{q}}-\frac{\beta_{0}}{4} c_{3}^{q \bar{q}}+C_{A} \frac{\beta_{0}}{8}\right] \mathcal{D}_{2}\left(s_{4}\right) \\
& \quad+\left\{c_{3}^{q \bar{q}} c_{1}^{q \bar{q}}+\left(c_{2}^{q \bar{q}}\right)^{2}-\zeta_{2}\left(c_{3}^{q \bar{q}}\right)^{2}-\frac{\beta_{0}}{2} T_{2}^{q \bar{q}}+\frac{\beta_{0}}{4} c_{3}^{q \bar{q}} \ln \left(\frac{\mu_{R}^{2}}{s}\right)+2 C_{F} K\right.
\end{aligned}
$$




$$
\begin{aligned}
& \left.+C_{A}\left[-\frac{K}{2}+\frac{\beta_{0}}{4} \ln \left(\frac{p_{T}^{2}}{s}\right)\right]-\frac{\beta_{0}^{2}}{16}\right\} \mathcal{D}_{1}\left(s_{4}\right) \\
& +\left\{c_{2}^{q \bar{q}} c_{1}^{q \bar{q}}-\zeta_{2} c_{2}^{q \bar{q}} c_{3}^{q \bar{q}}+\zeta_{3}\left(c_{3}^{q \bar{q}}\right)^{2}-\frac{\beta_{0}}{2} T_{1}^{q \bar{q}}+\frac{\beta_{0}}{4} c_{2}^{q \bar{q}} \ln \left(\frac{\mu_{R}^{2}}{s}\right)+G_{q \bar{q}}^{(2)}\right. \\
& +C_{F}\left[\frac{\beta_{0}}{4} \ln ^{2}\left(\frac{\mu_{F}^{2}}{s}\right)-K \ln \left(\frac{\mu_{F}^{2}}{s}\right)+K \ln \left(\frac{p_{T}^{2}}{s}\right)\right] \\
& \left.+C_{A}\left[\frac{\beta_{0}}{8} \ln ^{2}\left(\frac{p_{T}^{2}}{s}\right)-\frac{K}{2} \ln \left(\frac{p_{T}^{2}}{s}\right)\right]-\frac{\beta_{0}^{2}}{16} \ln \left(\frac{p_{T}^{2}}{s}\right)\right\} \mathcal{D}_{0}\left(s_{4}\right) \\
& +R^{q \bar{q} \rightarrow \gamma g} \delta\left(s_{4}\right) .
\end{aligned}
$$

Here $K=C_{A}\left(67 / 18-\pi^{2} / 6\right)-5 n_{f} / 9, \zeta_{2}=\pi^{2} / 6$, and $\zeta_{3}=1.2020569 \cdots$. The function $G_{q \bar{q}}^{(2)}$ denotes a set of two-loop contributions [8] and is given by

$$
G_{q \bar{q}}^{(2)}=C_{F} C_{A}\left(\frac{7}{2} \zeta_{3}+\frac{22}{3} \zeta_{2}-\frac{299}{27}\right)+n_{f} C_{F}\left(-\frac{4}{3} \zeta_{2}+\frac{50}{27}\right)
$$

We determine in the virtual corrections $R^{q \bar{q} \rightarrow \gamma g}$ only the terms that involve the renormalization and factorization scales, denoted as $R^{\mu q \bar{q} \rightarrow \gamma g}$ and given explicitly by

$$
\begin{aligned}
R^{\mu q \bar{q} \rightarrow \gamma g}= & \ln ^{2}\left(\frac{\mu_{F}^{2}}{p_{T}^{2}}\right)\left\{\frac{C_{F}^{2}}{2}\left[\frac{3}{2}+\ln \left(\frac{p_{T}^{2}}{s}\right)\right]^{2}-2 \zeta_{2} C_{F}^{2}+\frac{3}{16} \beta_{0} C_{F}+\frac{\beta_{0}}{8} C_{F} \ln \left(\frac{p_{T}^{2}}{s}\right)\right\} \\
+ & \ln \left(\frac{\mu_{F}^{2}}{p_{T}^{2}}\right) \ln \left(\frac{\mu_{R}^{2}}{p_{T}^{2}}\right) \frac{\left(-\beta_{0}\right)}{2} C_{F}\left[\frac{3}{2}+\ln \left(\frac{p_{T}^{2}}{s}\right)\right]+\ln ^{2}\left(\frac{\mu_{R}^{2}}{p_{T}^{2}}\right) \frac{\beta_{0}^{2}}{16} \\
+ & \ln \left(\frac{\mu_{F}^{2}}{p_{T}^{2}}\right)\left\{C_{F}^{2}\left[\frac{3}{2}+\ln \left(\frac{p_{T}^{2}}{s}\right)\right]^{2} \ln \left(\frac{p_{T}^{2}}{s}\right)-C_{F}\left[\frac{3}{2}+\ln \left(\frac{p_{T}^{2}}{s}\right)\right]\left[\frac{\beta_{0}}{4} \ln \left(\frac{p_{T}^{2}}{s}\right)+T_{1}^{q \bar{q}}\right]\right. \\
& -\frac{\zeta_{2}}{2} \beta_{0} C_{F}-C_{F} \frac{K}{2} \ln \left(\frac{p_{T}^{2}}{s}\right)+C_{F}^{2}\left(-11 \zeta_{3}+\frac{3}{2} \zeta_{2}-\frac{3}{16}\right) \\
& \left.+C_{F} C_{A}\left(\frac{7}{2} \zeta_{3}-\frac{11}{6} \zeta_{2}-\frac{17}{48}\right)+n_{f} C_{F}\left(\frac{\zeta_{2}}{3}+\frac{1}{24}\right)\right\} \\
+ & \ln \left(\frac{\mu_{R}^{2}}{p_{T}^{2}}\right)\left\{-\frac{\beta_{0}}{2} C_{F}\left[\frac{3}{2}+\ln \left(\frac{p_{T}^{2}}{s}\right)\right] \ln \left(\frac{p_{T}^{2}}{s}\right)+\frac{\beta_{0}}{2} T_{1}^{q \bar{q}}+\frac{\beta_{0}^{2}}{8} \ln \left(\frac{p_{T}^{2}}{s}\right)+\frac{\beta_{1}}{16}\right\}, \quad(2.10)
\end{aligned}
$$

where $\beta_{1}=34 C_{A}^{2} / 3-2 n_{f}\left(C_{F}+5 C_{A} / 3\right)$ and

$$
\gamma_{q / q}^{\prime(2)}=C_{F}^{2}\left(\frac{3}{32}-\frac{3}{4} \zeta_{2}+\frac{3}{2} \zeta_{3}\right)+C_{F} C_{A}\left(-\frac{3}{4} \zeta_{3}+\frac{11}{12} \zeta_{2}+\frac{17}{96}\right)+n_{f} C_{F}\left(-\frac{\zeta_{2}}{6}-\frac{1}{48}\right) .
$$

For the $q g \rightarrow \gamma q$ process we have

$$
{\hat{\sigma^{\prime}}}_{q g}^{(2)}=\frac{1}{2}\left(c_{3}^{q g}\right)^{2} \mathcal{D}_{3}\left(s_{4}\right)+\left[\frac{3}{2} c_{3}^{q g} c_{2}^{q g}-\frac{\beta_{0}}{4} c_{3}^{q g}+C_{F} \frac{\beta_{0}}{8}\right] \mathcal{D}_{2}\left(s_{4}\right)
$$




$$
\begin{aligned}
& +\left\{c_{3}^{q g} c_{1}^{q g}+\left(c_{2}^{q g}\right)^{2}-\zeta_{2}\left(c_{3}^{q g}\right)^{2}-\frac{\beta_{0}}{2} T_{2}^{q g}+\frac{\beta_{0}}{4} c_{3}^{q g} \ln \left(\frac{\mu_{R}^{2}}{s}\right)+\left(C_{F}+C_{A}\right) K\right. \\
& \left.+C_{F}\left[-\frac{K}{2}+\frac{\beta_{0}}{4} \ln \left(\frac{p_{T}^{2}}{s}\right)\right]-\frac{3}{16} \beta_{0} C_{F}\right\} \mathcal{D}_{1}\left(s_{4}\right) \\
& +\left\{c_{2}^{q g} c_{1}^{q g}-\zeta_{2} c_{2}^{q g} c_{3}^{q g}+\zeta_{3}\left(c_{3}^{q g}\right)^{2}-\frac{\beta_{0}}{2} T_{1}^{q g}+\frac{\beta_{0}}{4} c_{2}^{q g} \ln \left(\frac{\mu_{R}^{2}}{s}\right)+G_{q g}^{(2)}\right. \\
& +\left(C_{F}+C_{A}\right)\left[\frac{\beta_{0}}{8} \ln ^{2}\left(\frac{\mu_{F}^{2}}{s}\right)-\frac{K}{2} \ln \left(\frac{\mu_{F}^{2}}{s}\right)\right]+C_{F} K \ln \left(\frac{-t}{s}\right)+C_{A} K \ln \left(\frac{-u}{s}\right) \\
& \left.+C_{F}\left[\frac{\beta_{0}}{8} \ln ^{2}\left(\frac{p_{T}^{2}}{s}\right)-\frac{K}{2} \ln \left(\frac{p_{T}^{2}}{s}\right)\right]-\frac{3}{16} C_{F} \beta_{0} \ln \left(\frac{p_{T}^{2}}{s}\right)\right\} \mathcal{D}_{0}\left(s_{4}\right) \\
& +R^{q g \rightarrow \gamma q} \delta\left(s_{4}\right) .
\end{aligned}
$$

The function $G_{q g}^{(2)}$ denotes a set of two-loop contributions [8] and is given by

$$
\begin{aligned}
G_{q g}^{(2)} & =C_{F}^{2}\left(-\frac{3}{32}+\frac{3}{4} \zeta_{2}-\frac{3}{2} \zeta_{3}\right)+C_{F} C_{A}\left(\frac{3}{4} \zeta_{3}-\frac{11}{12} \zeta_{2}-\frac{189}{32}\right) \\
& +C_{A}^{2}\left(\frac{7}{4} \zeta_{3}+\frac{11}{3} \zeta_{2}-\frac{41}{216}\right)+n_{f} C_{F}\left(\frac{1}{6} \zeta_{2}+\frac{17}{16}\right)+n_{f} C_{A}\left(-\frac{2}{3} \zeta_{2}-\frac{5}{108}\right)
\end{aligned}
$$

Finally, the terms in $R^{q g \rightarrow \gamma q}$ that involve the renormalization and factorization scales, denoted as $R^{\mu q g \rightarrow \gamma q}$, are given explicitly by

$$
\begin{aligned}
& R^{\mu q g \rightarrow \gamma q}=\ln ^{2}\left(\frac{\mu_{F}^{2}}{p_{T}^{2}}\right)\left\{\frac{1}{2}\left[\frac{\beta_{0}}{4}+\frac{3}{4} C_{F}+C_{F} \ln \left(\frac{-t}{s}\right)+C_{A} \ln \left(\frac{-u}{s}\right)\right]^{2}-\frac{\zeta_{2}}{2}\left(C_{F}+C_{A}\right)^{2}\right. \\
&\left.+\frac{\beta_{0}}{8}\left[\frac{\beta_{0}}{4}+\frac{3}{4} C_{F}+C_{F} \ln \left(\frac{-t}{s}\right)+C_{A} \ln \left(\frac{-u}{s}\right)\right]\right\} \\
&+\ln \left(\frac{\mu_{F}^{2}}{p_{T}^{2}}\right) \ln \left(\frac{\mu_{R}^{2}}{p_{T}^{2}}\right) \frac{\left(-\beta_{0}\right)}{2}\left[\frac{\beta_{0}}{4}+\frac{3}{4} C_{F}+C_{F} \ln \left(\frac{-t}{s}\right)+C_{A} \ln \left(\frac{-u}{s}\right)\right]+\ln ^{2}\left(\frac{\mu_{R}^{2}}{p_{T}^{2}}\right) \frac{\beta_{0}^{2}}{16} \\
&+\ln \left(\frac{\mu_{F}^{2}}{p_{T}^{2}}\right)\left\{\frac{\left(\frac{\beta_{0}}{4}+\frac{3}{4} C_{F}+C_{F} \ln \left(\frac{-t}{s}\right)+C_{A} \ln \left(\frac{-u}{s}\right)\right]^{2} \ln \left(\frac{p_{T}^{2}}{s}\right)}{}-\frac{\beta_{0}}{2}+\frac{3}{4} C_{F}+C_{F} \ln \left(\frac{-t}{s}\right)+C_{A} \ln \left(\frac{-u}{s}\right)\right]-C_{F}^{2}\left(\frac{5}{2} \zeta_{3}+\frac{3}{32}\right)-C_{A}^{2}\left(\frac{11}{4} \zeta_{3}+\frac{2}{3}\right) \\
&\left.-C_{F} C_{A}\left(\frac{9}{4} \zeta_{3}+\frac{5}{3} \zeta_{2}+\frac{17}{96}\right)+n_{f} C_{F}\left(\frac{\zeta_{2}}{6}+\frac{7}{48}\right)+n_{f} \frac{C_{A}}{6}\right\} \\
&+\ln \left(\frac{\mu_{R}^{2}}{p_{T}^{2}}\right)\{-\left.\frac{\beta_{0}}{2}\left[\frac{3}{4} C_{F}+C_{F} \ln \left(\frac{-t}{s}\right)+C_{A} \ln \left(\frac{-u}{s}\right)\right] \ln \left(\frac{p_{T}^{2}}{s}\right)+\frac{\beta_{0}}{2} T_{1}^{q g}+\frac{\beta_{1}}{16}\right\},(2.14)
\end{aligned}
$$

where

$$
\gamma_{g / g}^{\prime(2)}=C_{A}^{2}\left(\frac{2}{3}+\frac{3}{4} \zeta_{3}\right)-n_{f}\left(\frac{C_{F}}{8}+\frac{C_{A}}{6}\right)
$$




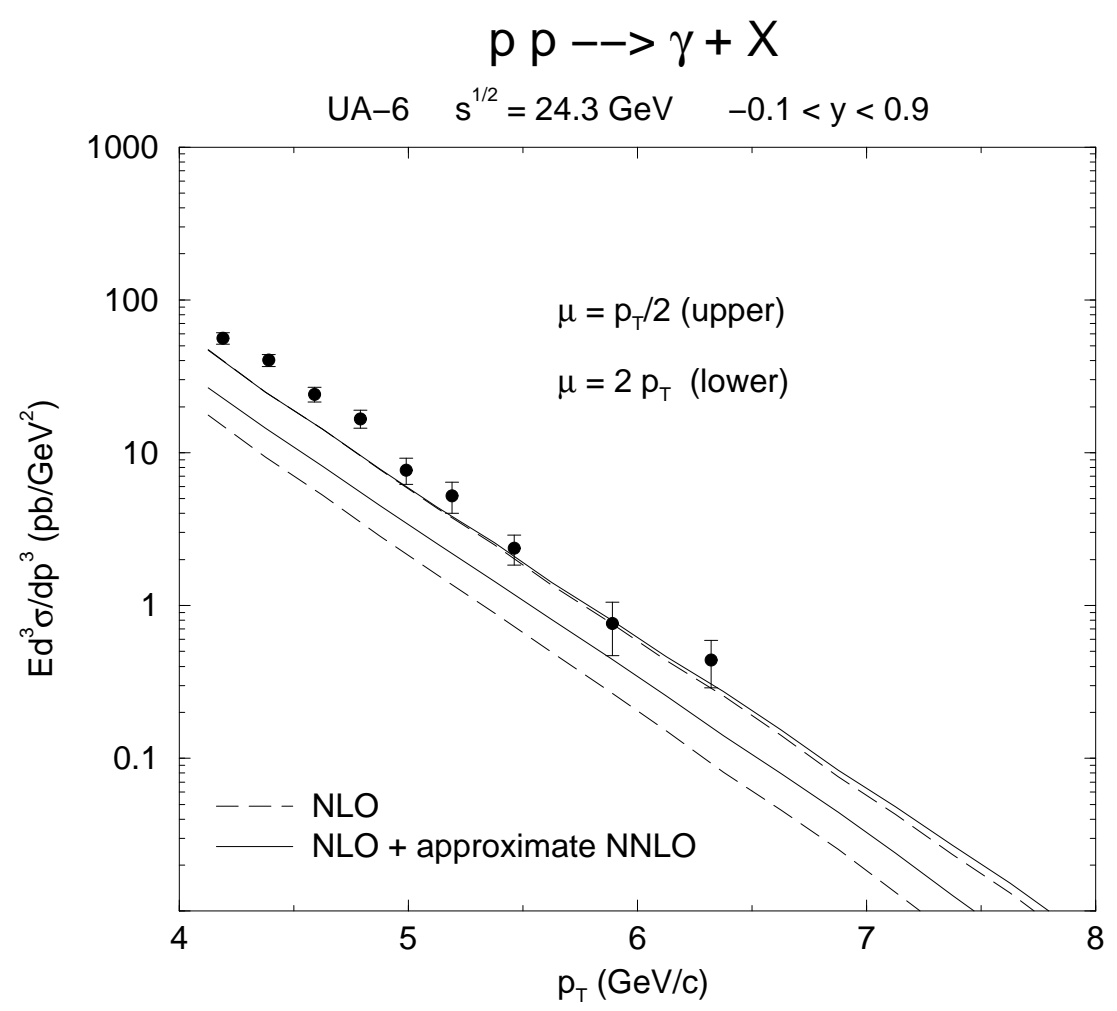

FIG. 1: NLO and NNLO results for direct photon production in hadronic collisions compared to $p p$ data from the UA-6 Collaboration [18] at $\sqrt{s}=24.3 \mathrm{GeV}$.

For both processes the coefficients of the $\mathcal{D}_{3}\left(s_{4}\right), \mathcal{D}_{2}\left(s_{4}\right)$, and $\mathcal{D}_{1}\left(s_{4}\right)$ terms were given previously in Ref. [5]. The additional subleading $\mathcal{D}_{0}\left(s_{4}\right)$ and $\delta\left(s_{4}\right)$ terms presented here are new.

\section{NUMERICAL RESULTS}

In order to show the effect of including the new NNLO terms, the same procedure employed in Ref. [5] has been used. First, a complete NLO calculation of the appropriate cross section is performed using a program [14] which employs the phase-space slicing technique described in Ref. [15]. The original NLO calculation has been extended to include a complete NLO treatment of the bremsstrahlung contribution. The Set 2 fragmentation functions of [16] have been used along with the CTEQ6M parton distribution functions [17]. In all cases the factorization and renormalization scales have been set equal to a common scale $\mu$ which has been chosen to be proportional to the photon's transverse momentum. Once the NLO results have been 


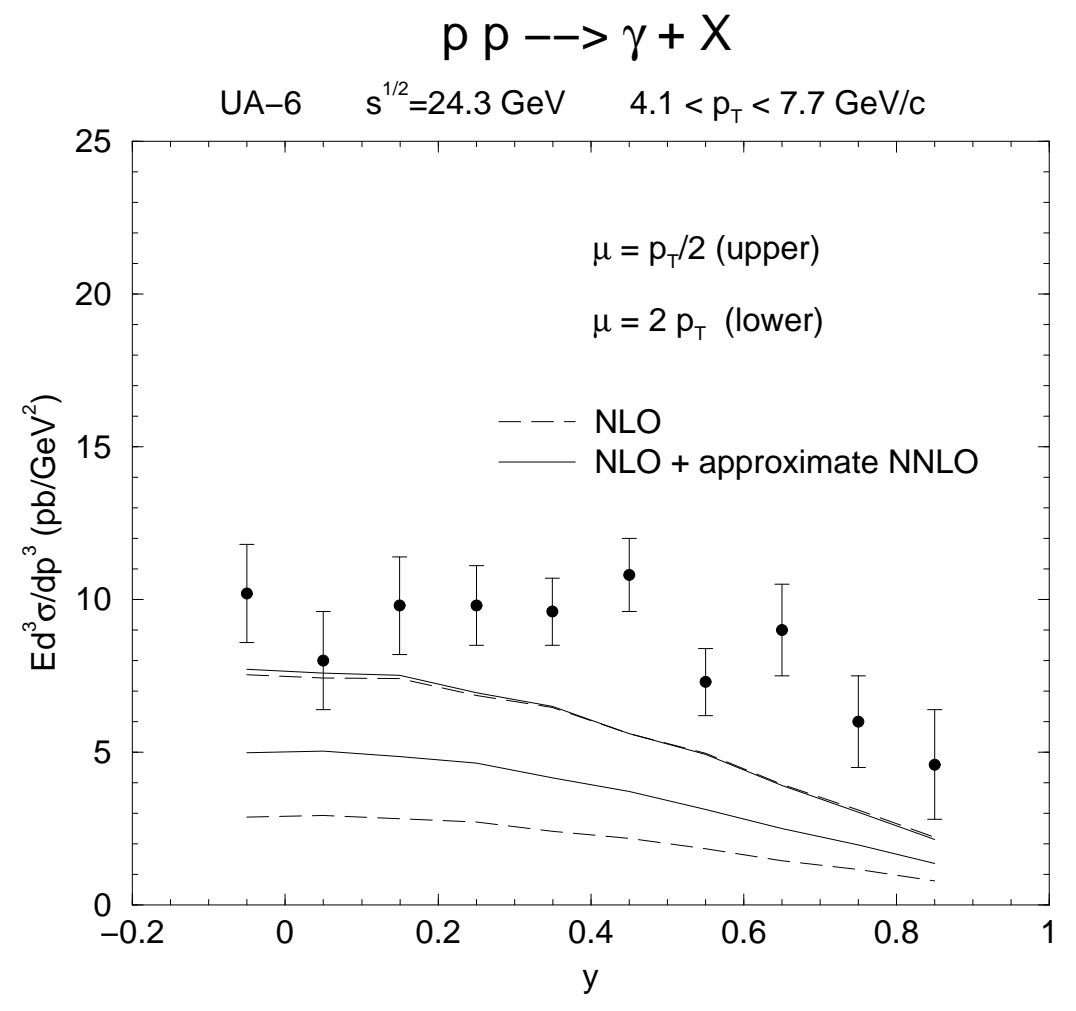

FIG. 2: NLO and NNLO results for direct photon production in hadronic collisions compared to $p p$ data for the rapidity distribution from the UA-6 Collaboration [18] at $\sqrt{s}=24.3 \mathrm{GeV}$.

obtained, the approximate NNLO contributions can be added to them. Several examples are discussed below, comparing the NLO and NLO + approximate NNLO results.

In Fig. 团 the NLO (dashed curves) and NLO plus approximate NNLO results (solid curves) are compared to data [18] from the UA-6 Collaboration for proton proton interactions. In each case the upper (lower) curve corresponds to the scale choice of $\mu=p_{T} / 2\left(2 p_{T}\right)$. The pattern demonstrated previously in Fig. 6 of Ref. [5] is still found to be true, even after the addition of the newly calculated terms presented in this work. The scale dependence of the NLO result is greatly decreased by the addition of the NNLO terms. Furthermore, one can see that for the scale choice of $\mu=p_{T} / 2$ the NNLO scale dependent terms give a negligible contribution. Even with the NNLO contributions, the results lie somewhat below the data at the lower values of $p_{T}$.

In Fig. 2 the rapidity dependence is shown for the UA-6 proton proton data. Again, the scale dependent NNLO terms give a negligible contribution for the choice $\mu=p_{T} / 2$ and the overall 


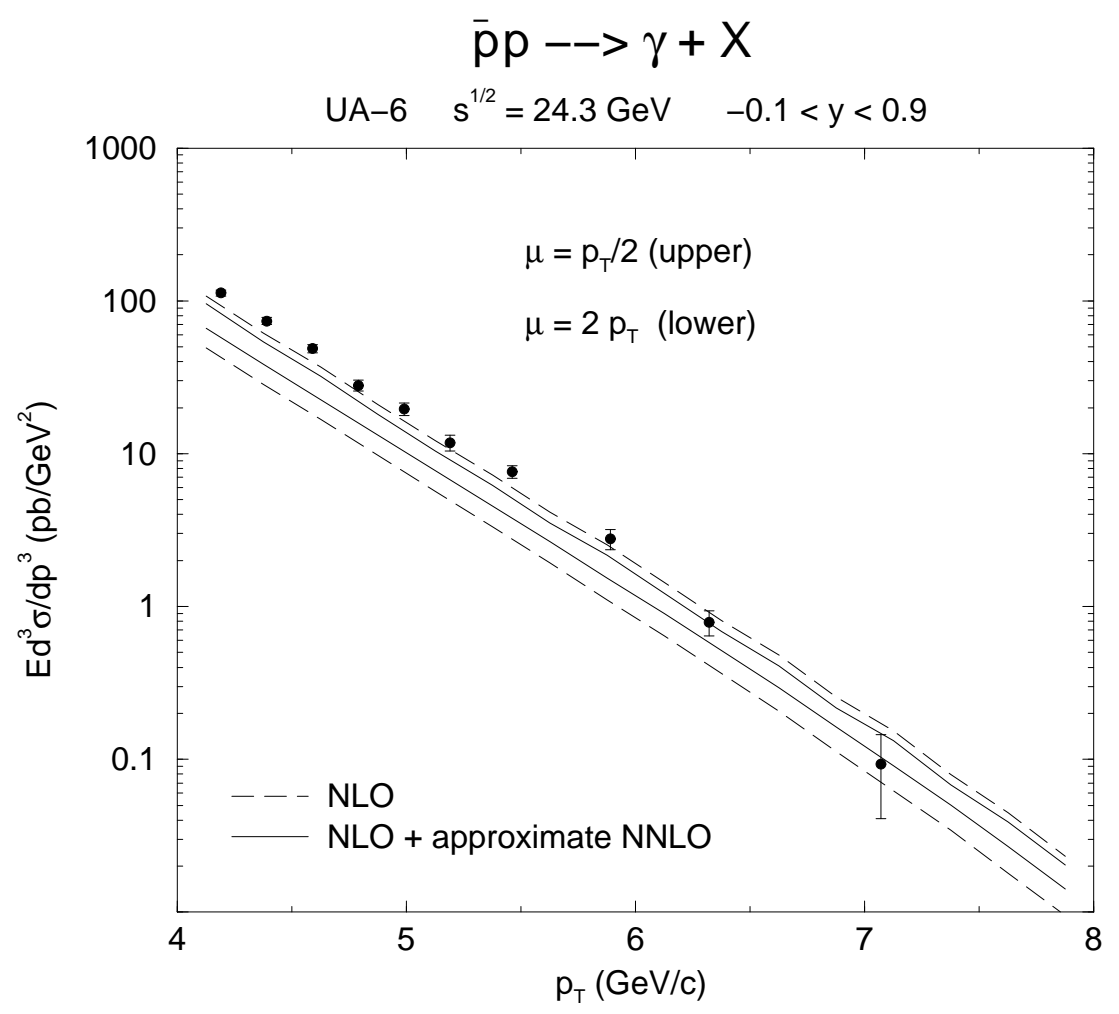

FIG. 3: NLO and NNLO results for direct photon production in hadronic collisions compared to $p \bar{p}$ data from the UA-6 Collaboration [18] at $\sqrt{s}=24.3 \mathrm{GeV}$.

scale dependence is greatly reduced when the NNLO terms are added. As noted previously, the curves lie below the data over the majority of the rapidity range shown. Of course, this distribution is dominated by the contributions from the low end of the $p_{T}$ range, so this is no surprise, given the results shown in Fig. 1.

In Fig. [3 the photon $p_{T}$ distribution is shown for the case of $p \bar{p}$ interactions and compared to data from the UA-6 Collaboration [18]. Whereas the $p p$ reaction is dominated by the $q g \rightarrow \gamma q$ subprocess, the $p \bar{p}$ reaction receives additional significant contributions from the $q \bar{q} \rightarrow \gamma g$ subprocess. Nevertheless, a pattern similar to that in the previous two figures is apparent here as well. Note, however, that for the case of $\mu=p_{T} / 2$, the NNLO contribution is negative, further reducing the scale dependence shown in Fig. 3. As for the $p p$ case, the band formed by the theoretical curves lies somewhat below the data at the lower values of $p_{T}$.

Finally, in Fig. [4 a similar comparison is made to data from the E-706 Collaboration [19]. The same behavior seen in the previous figures is evident here, although the theoretical band 


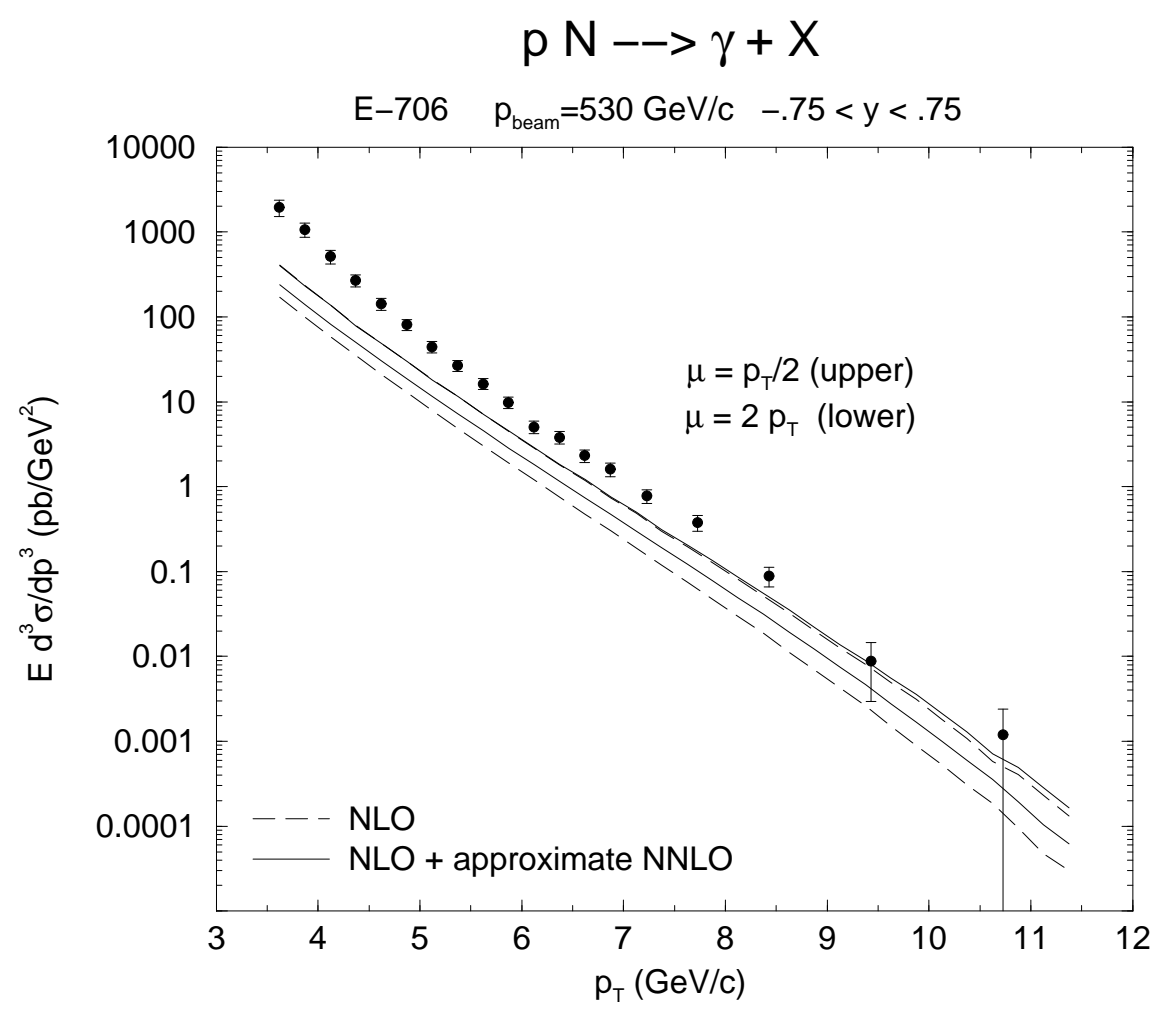

FIG. 4: NLO and NNLO results for direct photon production in hadronic collisions compared to data from the E-706 Collaboration [19] at $\mathrm{p}_{l a b}=530 \mathrm{GeV} / \mathrm{c}$.

now lies significantly below the data at the lower end of the range covered by the data.

\section{CONCLUSIONS}

We have extended the results of Ref. [5] to include additional NNLO subleading logarithms resulting from soft gluon corrections for direct photon production. The additional terms are numerically small and the results remain qualitatively the same as in the previous analysis. In particular, the reduced scale dependence relative to NLO calculations remains.

\section{Acknowledgments}

The research of N.K. has been supported by a Marie Curie Fellowship of the European Community programme "Improving Human Research Potential" under contract number HPMF-CT- 
2001-01221. The research of J.O. is supported in part by the U.S. Department of Energy.

[1] P. Aurenche, A. Douiri, R. Baier, M. Fontannaz, and D. Schiff, Phys. Lett. 140B, 87 (1984);

P. Aurenche, R. Baier, M. Fontannaz, and D. Schiff, Nucl. Phys. B297, 661 (1988).

[2] L.E. Gordon and W. Vogelsang, Phys. Rev. D 48, 3136 (1993).

[3] E. Laenen, G. Oderda, and G. Sterman, Phys. Lett. B 438, 173 (1998).

[4] S. Catani, M.L. Mangano, P. Nason, C. Oleari, and W. Vogelsang, JHEP 03, 025 (1999).

[5] N. Kidonakis and J.F. Owens, Phys. Rev. D 61, 094004 (2000).

[6] G. Sterman and W. Vogelsang, JHEP 02, 016 (2001).

[7] E. Laenen, G. Sterman, and W. Vogelsang, Phys. Rev. Lett. 84, 4296 (2000); Phys. Rev. D 63, 114018 (2001).

[8] N. Kidonakis, hep-ph/0303186.

[9] N. Kidonakis and G. Sterman, Phys. Lett. B 387, 867 (1996); Nucl. Phys. B505, 321 (1997).

[10] N. Kidonakis, G. Oderda, and G. Sterman, Nucl. Phys. B525, 299 (1998); B531, 365 (1998).

[11] N. Kidonakis and V. Del Duca, Phys. Lett. B 480, 87 (2000).

[12] N. Kidonakis, Int. J. Mod. Phys. A 15, 1245 (2000); Phys. Rev. D 64, 014009 (2001).

[13] N. Kidonakis and J.F. Owens, Phys. Rev. D 63, 054019 (2001).

[14] H. Baer, J. Ohnemus, and J.F. Owens, Phys. Rev. D 42, 61 (1990).

[15] B.W. Harris and J.F. Owens, Phys. Rev. D 65, 094032 (2002).

[16] L. Bourhis, M. Fontannaz, J.Ph. Guillet, and M. Werlen, Eur. Phys. J. C 19, 89 (2001).

[17] J. Pumplin, D.R. Stump, J. Huston, H.L. Lai, P. Nadolsky, and W.K. Tung, JHEP 07, 012 (2002).

[18] UA-6 Collaboration, G. Ballochi et al., Phys. Lett. B 436, 222 (1998).

[19] E-706 Collaboration, L. Apanasevich et al., Phys. Rev. Lett. 81, 2642 (1998). 